\title{
Essential role for Bclaf1 in lung development and immune system function
}

\author{
J Peter McPherson ${ }^{\star, 1}$, H Sarras ${ }^{1}$, B Lemmers ${ }^{2,3}$, L Tamblyn ${ }^{1}$, E Migon $^{2,3}$, E Matysiak-Zablocki ${ }^{2,3}$, A Hakem ${ }^{2,3}$, S Alizadeh Azami ${ }^{1}$, \\ R Cardoso ${ }^{2,3}$, J Fish ${ }^{2,3}$, O Sanchez ${ }^{4}$, M Post ${ }^{5}$ and R Hakem ${ }^{\star, 2,3}$
}

Bcl-2 associated factor 1 (Bclaf1) is a nuclear protein that was originally identified in a screen of proteins that interact with the adenoviral bcl-2 homolog E1B19K. Overexpression of Bclaf1 was shown to result in apoptosis and transcriptional repression that was reversible in the presence of $\mathrm{Bcl}-2$ or $\mathrm{Bcl}-\mathrm{x}_{\mathrm{L}}$. Furthermore, antiapoptotic members, but not proapoptotic members of the Bcl-2 protein family, were shown to interact with Bclaf1 and prevent its localization to the nucleus. Bclaf1 has also recently been identified as a binding partner for Emerin, a nuclear membrane protein that is mutated in X-linked recessive Emery-Dreifuss muscular dystrophy. To ascertain the in vivo function of Bclaf1, we have generated mice that carry a targeted mutation of the bclaf1 locus. In this study, we show that Bclaf1 is required for proper spatial and temporal organization of smooth muscle lineage during the saccular stage of lung development. We also show that Bclaf1 is dispensable for thymocyte development but is essential for peripheral T-cell homeostasis. Despite its postulated role as a proapoptotic protein, Bclaf1-deficient cells did not show any defect in cell death linked to development or after exposure to various apoptotic stimuli. Our findings show a critical role for Bclaf1 in developmental processes independent of apoptosis.

Cell Death and Differentiation (2009) 16, 331-339; doi:10.1038/cdd.2008.167; published online 14 November 2008

Apoptosis is a critical component of normal development and the cellular response to radio- and chemotherapy. ${ }^{1,2}$ Two distinct apoptotic pathways have been characterized: the extrinsic pathway triggered by transmembrane death receptors and the intrinsic pathway that signals through mitochondria. Members of the Bcl-2 protein family primarily impact the intrinsic pathway by controlling mitochondrial membrane permeability, the release of proapoptotic mitochondrial proteins, and caspase activation. ${ }^{1}$ Proapoptotic $\mathrm{Bcl}-2$ proteins such as Bak and Bax are activated directly after interactions with the 'BH3-only' Bcl-2 protein Bid. In addition, binding of other BH3-only proteins such as Noxa, Puma, Bad, and Bim to antiapoptotic $\mathrm{Bcl}-2$ proteins $\left(\mathrm{Bcl}-2\right.$ or $\mathrm{Bcl}-\mathrm{x}_{\mathrm{L}}$ ) results in activation of Bax and Bak. ${ }^{3-5}$

Bcl-2 associated factor 1 (Bclaf1; also known as Bcl-2associated transcription factor or Btf) was originally identified in a screen for adenoviral E1B19K protein partners. ${ }^{6}$ Subsequent studies with Bclaf1s, an alternatively-spliced form missing amino acid residues 797-846, showed interactions with $\mathrm{Bcl}-2$ and $\mathrm{Bcl}-\mathrm{x}_{\mathrm{L}}$. E1B19K, Bcl-2, and $\mathrm{Bcl}-\mathrm{x}_{\mathrm{L}}$ were found to sequester Bclaf1s to the nuclear periphery and cytoplasm. Ectopic Bclaf1 induced apoptosis as well as transcriptional repression, properties that were reversed in the presence of antiapoptotic Bcl-2 members. ${ }^{6}$ Although these findings implicate Bclaf1 in apoptotic signaling, Bclaf1 does not show structural similarities to Bcl-2 family members. Bclaf1 contains an N-terminal tract of Arg-Ser repeats (RS domain) that is typical of pre-mRNA processing factors and was identified in interchromatin granule clusters, nuclear substructures that appear to act as repositories for mRNA splicing and transcription factors. ${ }^{7,8}$ Emerin, a nuclear membrane protein that is mutated in X-linked Emery-Dreifuss muscular dystrophy, has also been found to interact with Bclaf1 as well as with several other proteins involved in transcription and pre-mRNA processing. ${ }^{9,10}$

Here, we show that Bclaf1 is critical for proper lung development and immune system homeostasis and function. Bclaf1 expression was found to be strongly upregulated during the saccular stage of murine embryonic lung development. Mice deficient in Bclaf1 predominantly die as neonates and exhibit defects in end-stage lung development that compromise survival. We also show that Bclaf1 is dispensable for thymocyte development and apoptosis but is essential for proper homeostasis of T- and B-cell lineages and activation-

\footnotetext{
${ }^{1}$ Department of Pharmacology, University of Toronto, Toronto, ON, Canada; ${ }^{2}$ Ontario Cancer Institute, University Health Network, Toronto, ON, Canada; ${ }^{3}$ Department of Medical Biophysics, University of Toronto, Toronto, ON, Canada; ${ }^{4}$ University of Ontario Institute of Technology, Oshawa, ON, Canada and ${ }^{5}$ Lung Biology Program, Hospital for Sick Children Research Institute, University of Toronto, Toronto, ON, Canada

${ }^{*}$ Corresponding authors: J Peter McPherson, University of Toronto, 1 King's College Circle, Toronto, Ontario, Canada M5S 1 A8.

Tel: + 416978 2727; Fax: + 416978 6395; E-mail: peter.mcpherson@utoronto.ca or

Razqallah Hakem, Department of Medical Biophysics, Ontario Cancer Institute/UHN, University of Toronto, 610 University Avenue PMH, Room 10-622, Toronto, Ontario, Canada M5G 2M9. Tel: + 416946 2398; Fax: + 416946 2984; E-mail: rhakem@uhnres.utoronto.ca

Keywords: lung development; smooth muscle; saccular stage; thymocyte development; apoptosis

Abbreviations: Bclaf1, Bcl-2 associated factor 1; ES cell, embryonic stem cell; E, embryonic day; PND, postnatal day; PCNA, proliferating cell nuclear antigen; SP-C, surfactant protein C; CCSP, Clara cell secretory 10-kDa protein; FACS, fluorescence-activated cell sorting; LNs, lymph nodes; BM, bone marrow; IL-2, interleukin 2; MEF, murine embryonic fibroblast

Received 25.7.08; revised 09.9.08; accepted 02.10.08; Edited by A Strasser; published online 14.11.08
} 
induced proliferation of $\mathrm{T}$ cells. Our findings do not support a role for Bclaf1 in regulating apoptosis during lung development or immune system function.

\section{Results}

Generation of Bclaf1-deficient mice and cells. Full-length cDNAs comprising the long and short isoforms of bclaf1 were isolated and sequenced, and a targeting vector was designed to replace the first three exons of bclaf1 with a neomycin-resistance cassette (including the exon encoding the predicted translation initiation start site) and insert a stop codon into the third exon (Figure 1a). The targeting vector was electroporated into $\mathrm{E} 14 \mathrm{~K}$ embryonic stem (ES) cells and three correctly targeted clones were obtained. Two ES clones contributed successfully to germline transmission. Homozygotes derived from both bclaf1 ${ }^{+/-}$ES clones had similar phenotypes in 129/C57BL/6 backgrounds. ES cell lines homozygous for the targeted disruption were generated by culturing bclaf1 $1^{+/-}$cells at an increased concentration of G418. Wild-type and mutant alleles were distinguished either by southern analysis (Figure 1b) or by PCR (not shown). Bclaf1 disruption results in the loss of expression of both the 5- and 3-kb bclaf1 mRNA species, as observed by northern analysis (Figure 1c). Western analysis of whole cell extracts using anti-Bclaf1 antiserum (Figure 1d) detected a single protein species migrating at $140 \mathrm{kDa}$ that was absent in extracts prepared from bclaf1 ${ }^{-/-}$cells, suggesting that the targeted disruption resulted in a null mutation. The apparent molecular weight $(140 \mathrm{kDa})$ was greater than that predicted from the Bclaf1 open reading frame (106 kDa), similar to results from an earlier study. ${ }^{10}$ Indirect immunofluorescence with affinity-purified anti-Bclaf1 (Figure 1e-f) showed nuclear localization of Bclaf1 with focal dot-like structures, as has been reported earlier. ${ }^{8,10}$ No Bclaf1 was detected in Bclaf1 ${ }^{-1-}$ murine embryonic fibroblasts (MEFs) (Figure 1g-h).

The murine bclaf1 locus is flanked by 2610016 C23Rik (separated by $5 \mathrm{~kb}$ ) and mtap7 (separated by $34 \mathrm{~kb}$ ) on chromosome 10. To assess whether expression of upstream or downstream genes was altered by the targeted disruption strategy, we quantified relative mRNA expression levels of Mtap7 and 26100162C23Rik from mRNA derived from wildtype and bclaf1 ${ }^{-1-}$ fibroblasts by quantitative real-time PCR using primers as described in Materials and Methods. We observed modest changes in expression in that a 1.10 \pm 0.15 fold increase (mean \pm S.D., $n=3$ ) in expression of Mtap7 mRNA and a $1.22 \pm 0.17$ increase (mean \pm S.D., $n=3$ ) in expression of $26100162 \mathrm{C} 23 \mathrm{Rik}$ in bclaf1 $^{-1-}$ versus wild type was detected when normalized to glyceraldehyde-3-phosphate dehydrogenase (GAPDH) expression. Although the function of the product of the 26100162C23Rik locus is unknown in mice, Mtap7 is known to be a microtubule-binding protein required for spermatogenesis in mice. ${ }^{11}$ Mice homozygous for this gene-trapped mtap7 allele are otherwise normal and viable. Taken together, the observed phenotypes of bclaf1 $1^{-1-}$ mice are unlikely to be attributable to the upstream or downstream effects on gene expression by the targeting strategy employed.
Bclaf1 is required for postnatal viability and end-stage lung development. Homozygous mutation of bclaf1 did not confer embryonic lethality; however, the majority of bclaf1 ${ }^{-/-}$ (approximately 95\%) die 24-48 h after birth and do not appear to suckle as evidenced by the lack of milk in the stomach despite the proper palate formation (Figure 1i). The other $5 \%$ of $b c l a f 1^{-1-}$ mice survive to approximately 3 weeks of age, whereupon they died. These mice were runted in appearance and size (Figure 1j), and $50 \%$ of these mice (5 out of 10) presented with polydactyly on the right hindpaw (Figure 1k).

To investigate the cause of perinatal lethality, histological analyses were performed on wild-type and Bclaf1-deficient mice at embryonic days 15.5-18.5 (E15.5-18.5) and postnatal day 1 (PND1). Lungs from newborn Bclaf1-deficient mice showed striking defects in lung morphology with features of arrested development of terminal air sacs together with mesenchymal thickening when compared with wild-type siblings (Figure 2a). The morphology of wild-type and bclaf1 $^{-1-}$ lungs at E15.5 to E18.5 was normal, consistent with a defect that manifested during the saccular stage of lung development (data not shown).

To determine whether the developmental defect in bclaf1 ${ }^{-1}$ lungs could be ascribed to inappropriate cell proliferation, we examined the abundance and sites of proliferating cells in lungs of three sibling sets of wild-type or Bclaf1deficient mice, either by BrdU labeling (for E16.5-E18.5 lungs) or by immunodetection of proliferating cell nuclear antigen (PCNA) in PND1 lungs. No significant difference in total cell proliferative index (mean \pm S.D. percentage of BrdU-positive cells, $n=3$ ) was observed in bclaf1 ${ }^{-/}$ lungs compared with wild type at E16.5 (15.3 $\pm 7.1 \%$ compared with $20.1 \pm 3.1 \%)$, at E17.5 (8.5 $\pm 1.7 \%$ compared with $9.2 \pm 1.5 \%)$, or at E18.5 $(7.3 \pm 3.8 \%$ compared with $5.3 \pm 1.5 \%$ ). Furthermore, at PND1, no significant difference in total cell proliferative index (mean \pm S.D. percentage of PCNA-positive cells, $n=4$ ) was observed in bclaf1 $^{-1-}$ lungs compared with wild type $(33.0 \pm 9.6 \%$ compared with $31.2 \pm 13.7 \%)$

To assess whether the bclaf1 ${ }^{-/}$lung defect could be ascribed to attenuated cell death, we analyzed lungs of three sibling sets of wild-type or bclaf1 ${ }^{-/}$mice at PND1 for apoptosis either by TUNEL staining or by immunostaining with an antibody against cleaved (activated) form of caspase 3 , a marker of apoptosis. In all sections examined from E16.5, E17.5, E18.5, and PND1 mice, the low frequency of apoptotic cells (less than $0.5 \%$ of cells were found to be positive for cleaved caspase 3 ) in lung tissue, regardless of developmental age or genotype, precluded any meaningful quantitative comparison.

Bclaf1 deficiency resulted in a sudden developmental arrest during end-stage lung development by PND1, yet bclaf1 ${ }^{-/-}$ lungs from earlier developmental stages were morphologically identical to sibling wild-type controls. To determine whether this particular requirement for Bclaf1 in the saccular stage was reflected in the expression profile of Bclaf1 protein during lung development, Bclaf1 expression was analyzed in wild-type E16.5-PND1 lungs by immunohistochemistry and indirect immunofluorescence (Figure $2 b$ and $c$ ). A dramatic increase in Bclaf1 expression was observed to occur between 


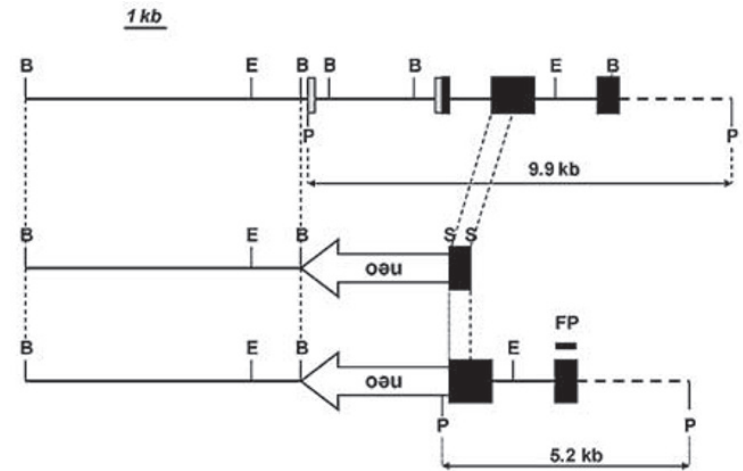

b $-/-+/-$ wt +/- wt wt +/-

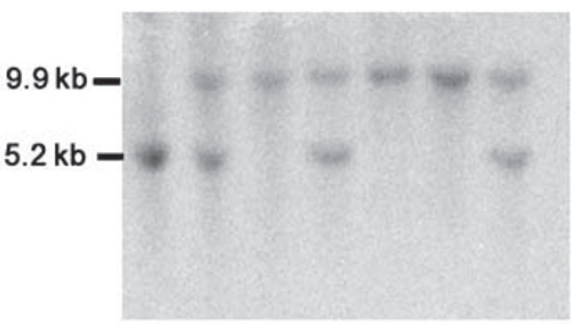

c
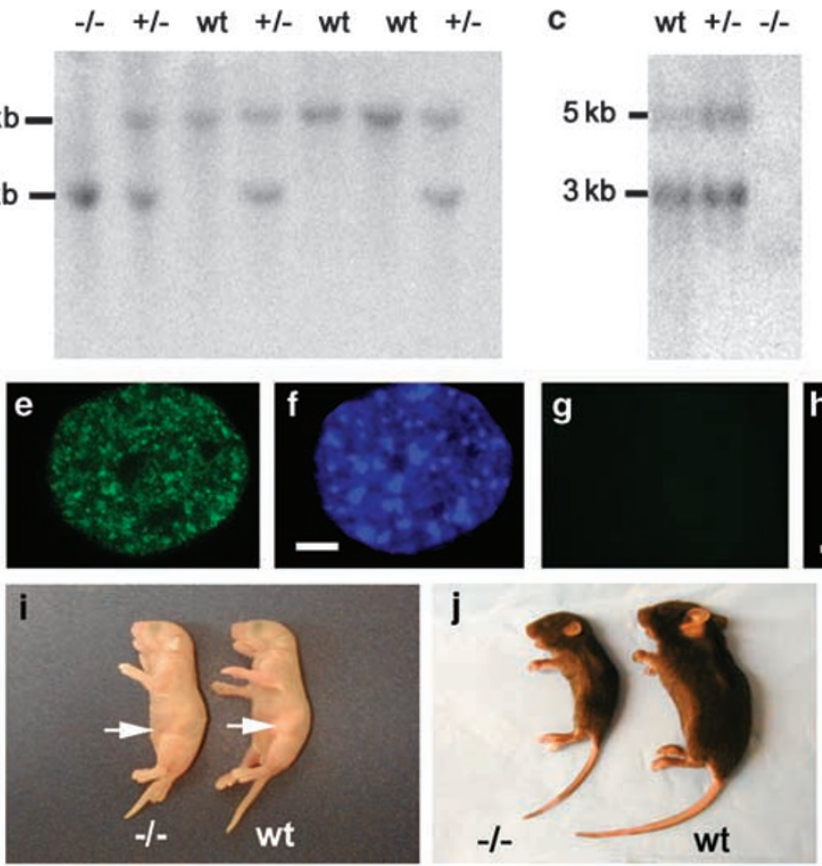

wild-type locus

targeting vector

targeted locus
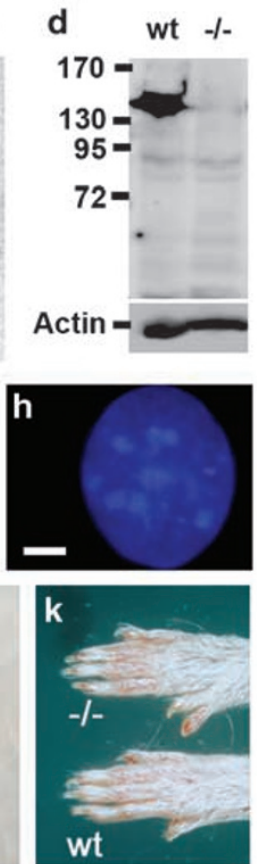

Figure 1 Targeted disruption of the bclaf1 locus and morphological characteristics of bclaf1 ${ }^{-1-}$ mice. (a) Strategy for targeted disruption of the $5^{\prime}$ end of the $b c l a f 1$ locus. Non-coding and coding exons are denoted as open and shaded boxes, respectively. The position and transcriptional orientation of the neomycin resistance gene (neo) are denoted by the open arrow. The position of sequence used as the $3^{\prime}$-flanking probe to distinguish wild-type and mutant alleles after Pstl digestion is denoted by FP. E, EcoRl; B, BamHI; H, Hindll. (b) Southern analysis of genomic DNA digested with Pstl from newborn littermates. (c) Northern analysis of total mRNA from fibroblasts derived from

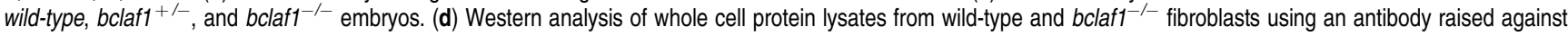
mouse Bclaf1. The blot was stripped and subsequently examined for actin expression to ensure equivalent protein loading (lower panel). (e-h) Representative photomicrographs of wild-type $(\mathbf{e}, \mathbf{f})$ _and $b c l a f 1^{-1-}(\mathbf{g}, \mathbf{h})$ fibroblasts subjected to indirect immunofluorescence using anti-Bclaf1 antibody (e, g). DAPI counterstaining of nuclear DNA is shown in (f, $\mathbf{h}$ ). (i) Newborn morphology of littermates from $b c l a f 1^{+/}$crosses. The majority of offspring that are bclaf1 ${ }^{-1-}$ fail to nurse (arrow: lack of milk in stomach) and die $24-48 \mathrm{~h}$ after birth. (j) A small percentage of bclaf1 $1^{-1-}$ mice survive up to 3 weeks of age, but are runted and die of unknown causes. (k) Roughly $50 \%$ of these surviving $b c l a f 1^{-1-}$ mice were observed to display polydactyly of the right hindpaw

E16.5-E17.5, which mirrors the transition in lung development from the canalicular phase to the saccular phase. ${ }^{12}$ When analyzed by indirect immunofluorescence, focal nuclear staining was evident in all cells of the lung but was especially prominent in epithelial cells lining the airways (Figure 2c).

Lineage-specific defects in lung maturation in bclaf1 ${ }^{-1-}$ neonates. To evaluate whether Bclaf1-deficient lungs had defects in differentiation, we evaluated the proportion and distribution of cell lineages in E17.5 and PND1 lungs from wild-type and bclaf1 $^{-/-}$siblings. Bclaf1 $1^{-/}$and wild-type lungs showed equivalent expression and spatial distribution of surfactant protein C (proSP-C, alveolar type II pneumocyte marker), T1- $\alpha$ (type I epithelial cell marker), Clara cell secretory 10-kDa protein (CCSP, bronchial epithelial cell marker), and the vascular cell marker Pecam1 (Figure 3 and data not shown). Interestingly, although equivalent at E17.5, smooth muscle $\alpha$-actin staining was markedly increased in bclaf1 ${ }^{-1-}$ lungs at PND1 compared with wild type (Figure $3 d-f$ ). The increased expression was most apparent at the periphery where it is normally not present and suggests a 
a

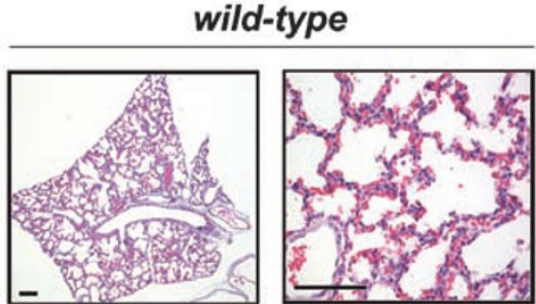

b

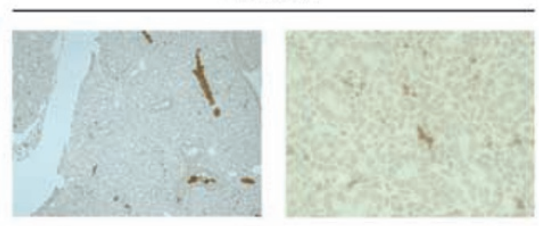

E17.5

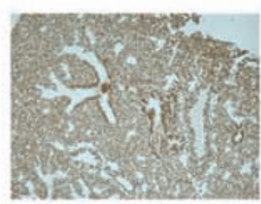

C
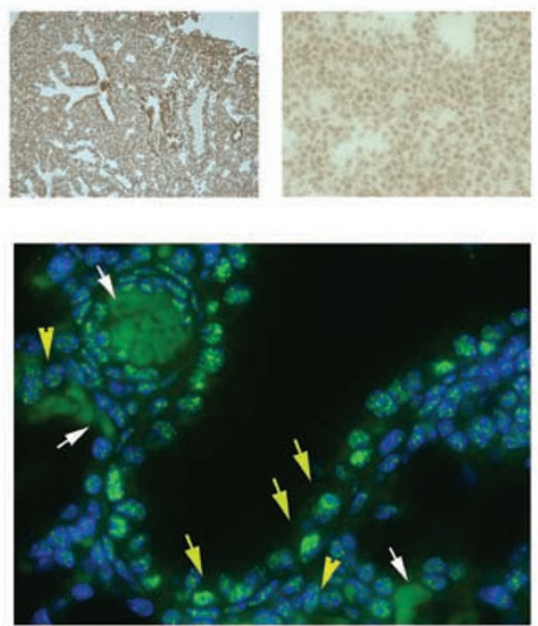

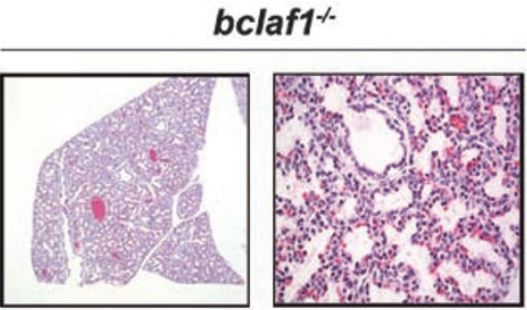

E18.5

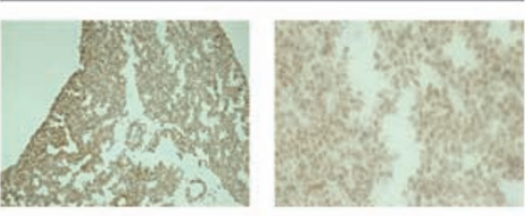

PND1
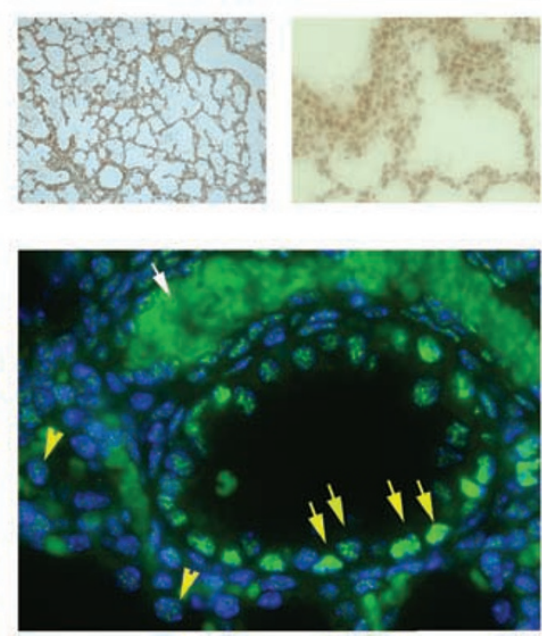

Figure 2 Developmental defects in lung maturation of bclaf1 ${ }^{-/}$mice and Bclaf1 expression during lung development. (a) Representative histological sections from postnatal day 1 wild-type and bclaf1 ${ }^{-1-}$ siblings after staining with hematoxylin and eosin. Bar: $100 \mu \mathrm{m}$. (b, c). Bclaf1 expression in fetal lung. (b) Representative histological sections from E16.5-PND1 wild-type lungs following staining with antibodies against Bclaf1 and visualized by DAB reagent. Bars: 200 and $40 \mu \mathrm{m}$. (c) Bclaf1 protein detected by indirect immunofluorescence using anti-Bclaf1 antibody and anti-rabbit-Cy5 (colored green) and counterstained with DAPI (blue). Yellow arrows, yellow arrowheads, and white arrows point to representative nuclei of airway epithelial cells, nuclei of mesenchyme cells, and autofluorescent red cells, respectively $(\times 63$ magnification)

defect in pulmonary mesenchymal differentiation in bclaf1 ${ }^{-/-}$ neonates.

Role of Bclaf1 in B- and T-cell lineage development. Bclaf1 appeared to be ubiquitously expressed in cells comprising the thymus and spleen when analyzed by immunohistochemistry (Supplementary Figure S1). To explore the in vivo role of Bclaf1 during lymphoid development or activation, we reconstituted irradiated Rag $^{-1-}$ mice, which lack $\mathrm{T}$ and $\mathrm{B}$ cells, with wild-type, bclaf1 $^{+/-}$, and bclaf1 ${ }^{-/-}$lymphoid progenitors. Fetal liver cells collected from bclaf1 ${ }^{-1-}$, bclaf1 ${ }^{+/-}$, or wild-type E15.5 embryos were injected intravenously into Rag2 $^{-1}$ mice. We used fetal liver cell suspensions from E15.5 embryos as a source of progenitors, because at this stage of development wild-type and bclaf1 ${ }^{-1-}$ embryos were phenotypically normal and at the expected Mendelian frequency. Four weeks postinjection, the representation of donor-derived thymocytes and peripheral $\mathrm{T}$ and $\mathrm{B}$ cells in the reconstituted Rag2 ${ }^{-1}$ recipient mice were determined to assess the effect of the bclaf1 mutation on lymphocyte development, survival, and homeostasis. Fluorescence-activated cell sorting (FACS) analyses were performed on thymocytes, splenocytes, lymph nodes (LNs), and bone marrow (BM) cells stained with monoclonal antibodies (mAbs) specific for markers of the different $\mathrm{T}$ - and B-lymphocyte subpopulations. No differences in cell numbers of various thymocyte subsets and TCR- $\alpha \beta$ expression were observed among Rag $^{-1-}$ mice reconstituted with bclaf1 $1^{-1-}$, bclaf1 ${ }^{+/-}$, or wild-type fetal liver cells (Figure 4a). The absolute number (mean \pm S.D., $n=4$ ) of thymocytes from $R$ Rag2 ${ }^{-1-}$ reconstituted mice was found to be $1.08 \pm 0.3 \times 10^{8}, 1.16 \pm 0.58 \times 10^{8}$, and $1.15 \pm 0.33 \times 10^{8}$ for wild type, bclaf1 $1^{+/}$, and bclaf1 $^{-1-}$, respectively. Similarly, the absolute number of splenocytes was $6.89 \pm 1.82 \times 10^{7}$ for wild type, $8.95 \pm 3.61 \times 10^{7}$ for bclaf1 $^{+/-}$, and $7.01 \pm 2.07 \times 10^{7}$ for bclaf1 ${ }^{-/-}$. However, a 

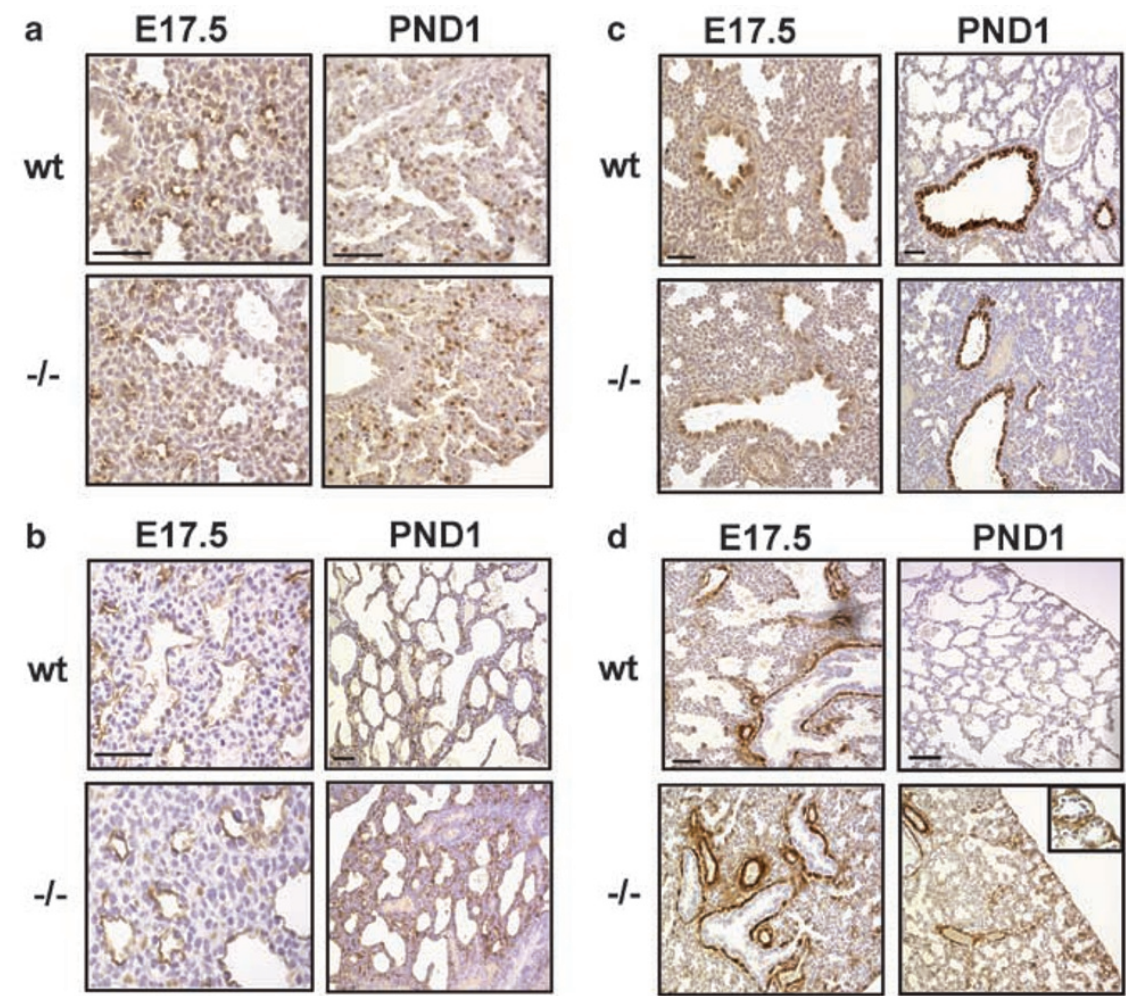

PND1
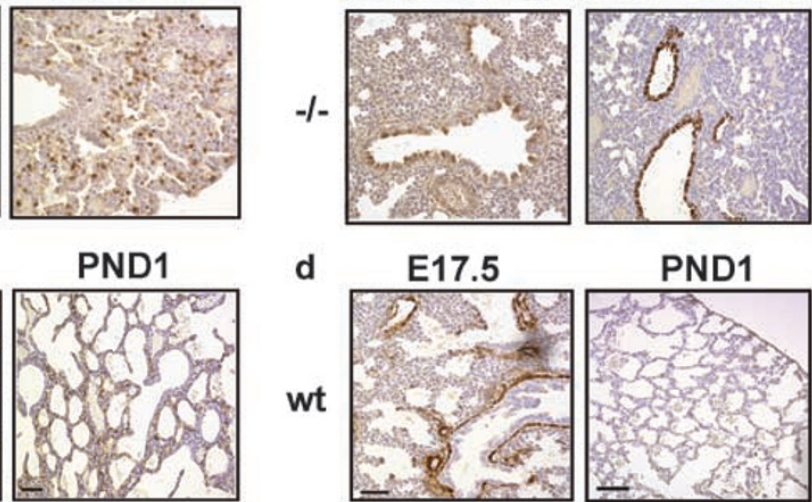

d
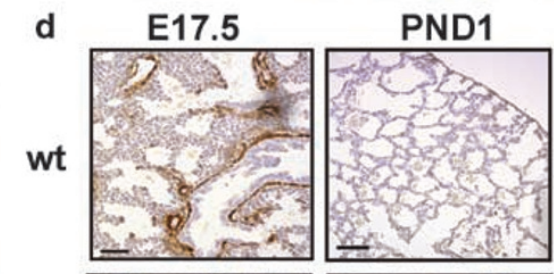

e
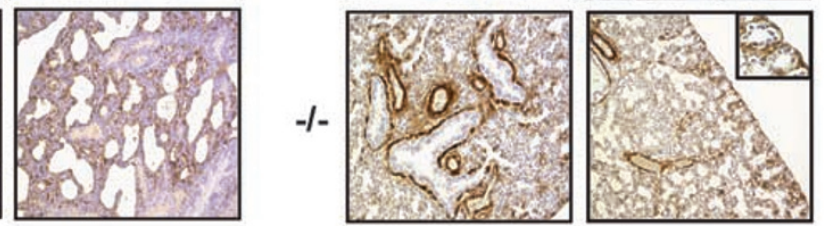

wt
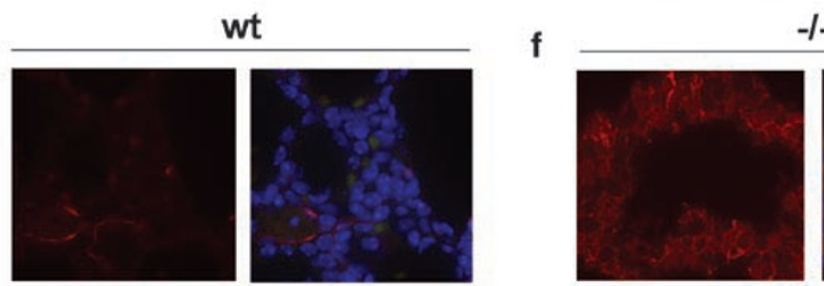

$-1-$

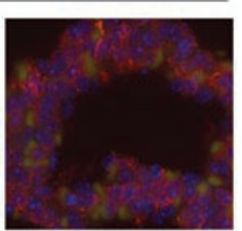

Figure 3 Expression of developmental markers in $b c l a f 1^{-/}$lungs. Representative histological sections from E17.5 and PND1 wild-type (wt) and bclaf1 ${ }^{-/-}(-/-)$siblings after staining with antibodies against proSP-C (a), CCSP (b), T1- $\alpha(\mathbf{c})$, and smooth muscle $\alpha$-actin (d). Bar: $50 \mu \mathrm{m}$. (e, f) Indirect immunofluorescence of smooth muscle $\alpha$-actin expression in PND1 (e) wild-type and (f) bclaf1 ${ }^{-1-}$ lungs. Slides were counterstained with DAPI to visualize nuclei (right panel). Green cells are autofluorescent red cells $(\times 63$ magnification)

marked difference in splenocytes was apparent after FACS analysis of Thy1- and B220-stained cell populations (Figure 4b, Supplementary Table S1). Despite the equivalent total cellularity in all reconstituted mice, the relative number of bclaf1 $^{-1-}$ Thy1-positive cells was drastically decreased, whereas the relative number of bclaf1 $^{-1-}$ B220-positive cells was markedly increased. Compared with populations of splenic CD4 + CD8- T cells, the population of CD4-CD8 + T cells was observed to be drastically reduced in $\mathrm{Rag}^{-1-}$ reconstituted with bclaf1 $^{-1-}$ or bclaf $^{+/-}$E14.5 fetal liver cells (Supplementary Table S1). Similar decreased representation of $\mathrm{T}$ cells compared with B-cells was also observed in $L N$ and in peripheral blood (Figure 4b). Thus, Bclaf1 deficiency appears to not only increase steady-state numbers of peripheral B cells but also compromises steady-state numbers of peripheral $\mathrm{T}$ cells leading to defective $\mathrm{T}$ - and B-cell homeostasis.

Response of Bclaf1-deficient cells to death stimuli. As Bclaf1 has originally been identified as a proapoptotic molecule that interacted with members of the antiapoptotic members of the $\mathrm{Bcl}-2$ protein family, we investigated the response of wild-type, bclaf1 ${ }^{+/}{ }^{-}$, and bclaf1 $1^{-/}$thymocytes, splenocytes, and activated peripheral $\mathrm{T}$ cells to a range of chemical agents that trigger apoptosis. Compared with thymocytes from $E \mu-b c l-2$ transgenic mice, which are resistant to a wide range of apoptotic stimuli, wild-type, bclaf1 ${ }^{+/-}$, and bclaf1 $^{-/-}$thymocytes all showed normal induction of programmed cell death following exposure to etoposide, CD8-FasL, staurosporine, dexamethasone, and $\gamma$-irradiation (Figure 5a). Similarly, no difference in cell death

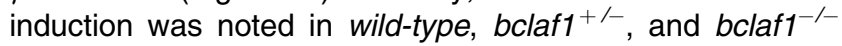
splenocytes treated with anisomycin, etoposide, staurosporine (Figure 5b), $\gamma$-irradiation, and dexamethasone (data not shown). Furthermore, no differences in apoptosis were observed when wild-type, bclaf1 ${ }^{+/}$, and bclaf1 ${ }^{-/}$ES cells were exposed to similar conditions (data not shown). Purified wild-type, bclaf1 ${ }^{+/}$, or bclaf1 ${ }^{-1-} \mathrm{T}$ and $\mathrm{B}$ cells, either untreated or induced to proliferate, showed no apparent defect in apoptosis (Supplementary Table S2). Hence, 

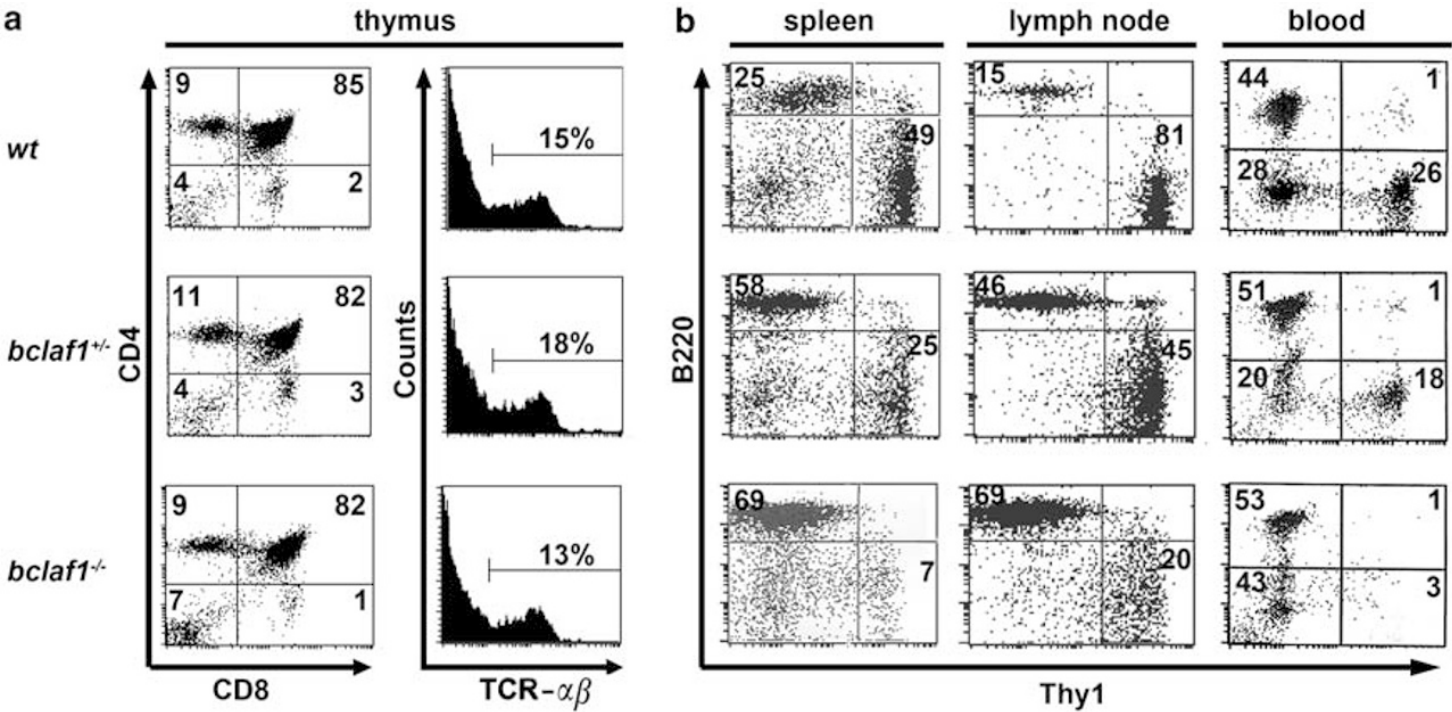

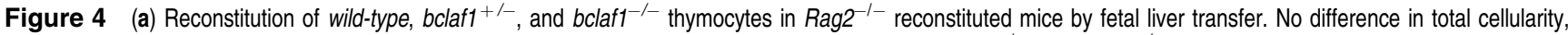
proportion of thymocyte subsets, or TCR- $\alpha \beta$ expression was observed. (b) Reconstitution of wild-type, bclaf1 ${ }^{+/-}$, and bclaf1 ${ }^{-1-}$ peripheral B (denoted by B220 staining) and T cells (denoted by Thy1 staining) in Rag2 ${ }^{-1-}$ reconstituted mice by fetal liver transfer. The bclaf1 ${ }^{-1-}$ reconstituted mice show a decreased proportion of peripheral T cells in the spleen, lymph nodes (LNs), and whole blood

a
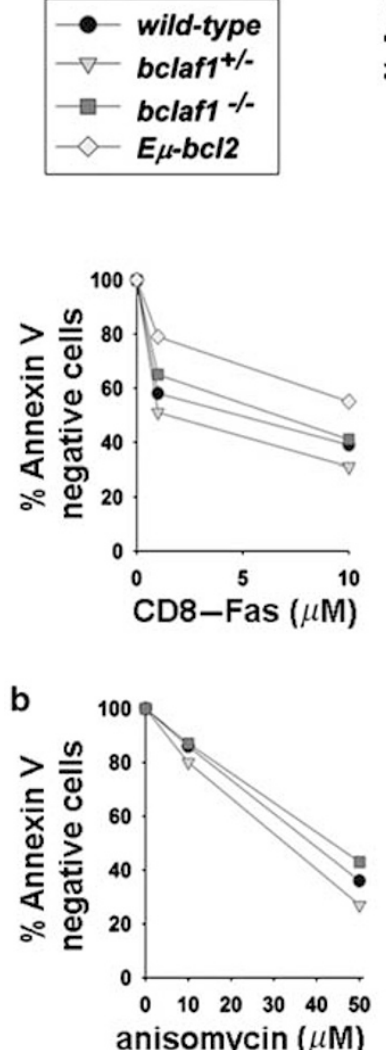
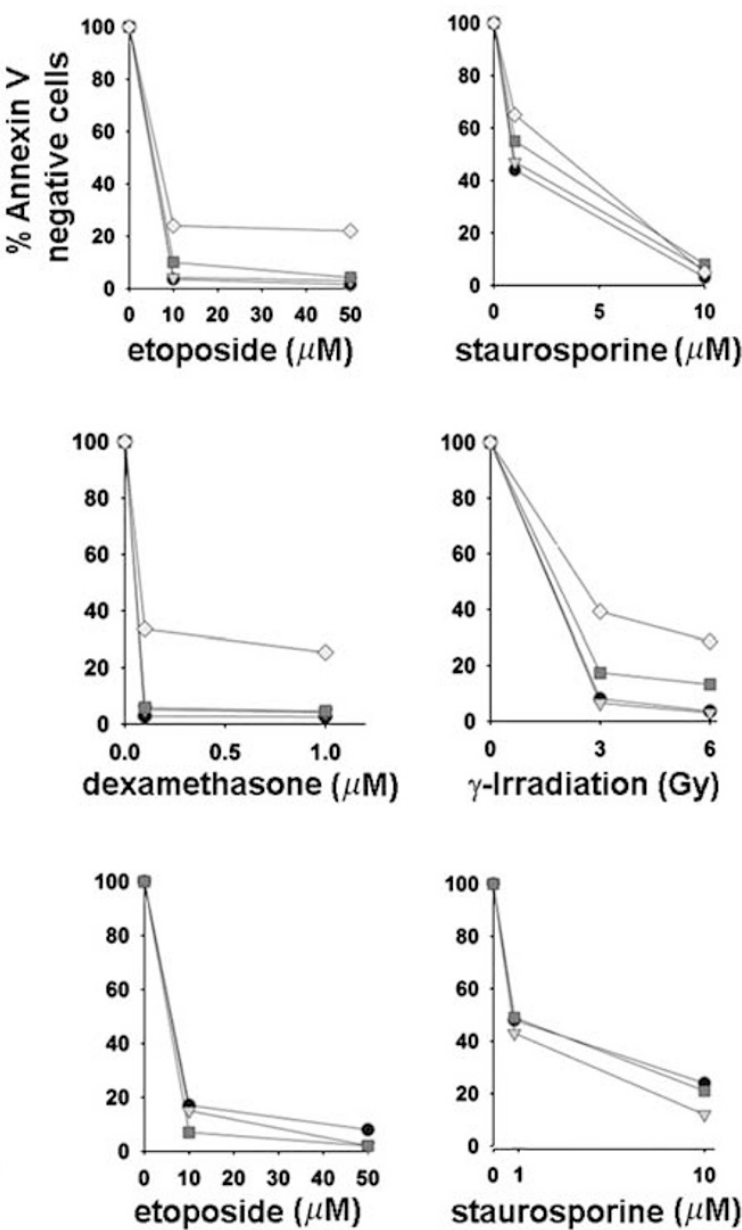

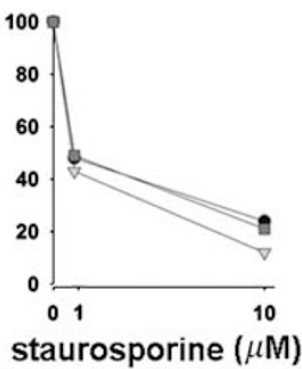

Figure 5 Bclaf1 is dispensable for apoptosis in thymocytes and splenocytes. (a) Apoptosis induced by etoposide, staurosporine, CD8-FasL, dexamethasone, and $\gamma$-irradiation in thymocytes from wild-type, bclaf1 ${ }^{+/}$, and $b c l a f 1^{-/}$reconstituted mice. Apoptosis in thymocytes from $\mathrm{E} \mu$-bcl-2 transgenic mice is included as positive controls. (b) Apoptosis induced by anisomycin, etoposide, and staurosporine in splenocytes from wild-type, bclaf1 ${ }^{+/}$, and $b c l a f 1^{-1-}$ reconstituted mice 
despite its physical interaction with antiapoptotic members of the Bcl-2 family, Bclaf1 appears to be dispensable for apoptosis in the context of the cell lineages and apoptotic stimuli examined.

Role of Bclaf1 in T-cell activation. To analyze the effect of Bclaf1 deficiency on proliferation, T-cell activation was analyzed in the presence of either anti-CD3 $\varepsilon$ and anti-CD28 or anti-CD $3 \varepsilon$ and interleukin 2 (IL-2), and B-cell activation was analyzed in the presence of either anti-IgM and antiCD40 or anti-IgM and IL-4. Peripheral T cells lacking Bclaf1 showed a lack of proliferation in the presence of either stimuli, compared with wild-type cells, as measured by $\left[{ }^{3} \mathrm{H}\right]$ thymidine incorporation (Figure 6a). Interestingly, bclaf1 $^{+1-} \mathrm{T}$ cells showed an intermediate proliferative response in response to either stimulus. In contrast, B cells lacking Bclaf1 did not show impaired proliferation compared with wild-type cells (Figure 6b). Cell cycle analyses of Thy1-positive cells following anti-CD3 and IL-2 activation showed a marked decrease in the percentage of bclaf1 ${ }^{-1-}$ $\mathrm{T}$ cells in the S-phase and their block at the $\mathrm{G} 1$ and $\mathrm{G} 2 / \mathrm{M}$ phases of the cell cycle as compared with bclaf1 ${ }^{+/-}$ cells (Figure $6 c$ ). These findings show that Bclaf1 is critical for proper proliferation and activation of peripheral T lymphocytes.
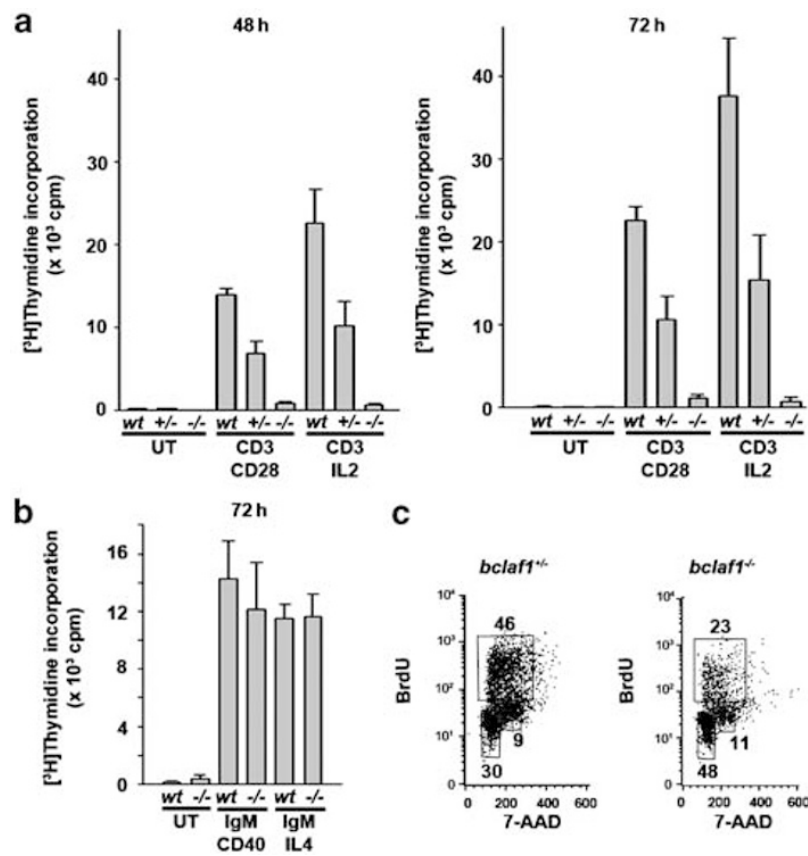

C
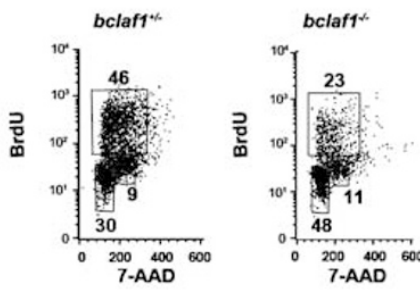

Figure 6 Peripheral bclaf $1^{-1-} \mathrm{T}$ cells show an activation-dependent proliferation defect. (a) Proliferation of purified T-cell (a) and B-cell (b) populations from bclaf1 ${ }^{+/-}$and $b c l a f 1^{-/}$reconstituted mice was assessed using $\left[{ }^{3} \mathrm{H}\right]$ thymidine incorporation after no activation stimulus (untreated, UT) or after stimulation with anti-CD3 $\varepsilon+$ anti-CD28 or with anti-CD3 $\varepsilon+$ IL-2 in the case of T cells, or with anti-IgM + anti-CD40 or anti-lgM + IL-4 in the case of B cells. Cells were assayed 48 and $72 \mathrm{~h}$ after stimulation for T cells or $72 \mathrm{~h}$ after stimulation for $B$ cells. (c) Cell cycle entry (BrdU incorporation) on Thy1-gated populations of CD3IL2 activated cells showing a decreased percentage of the S-phase and increased proportion of G1 and G2/M bclaf1 ${ }^{-/-}$activated cells

\section{Discussion}

Bclaf1 was initially described as a regulator of apoptosis and as an interacting partner with Bcl-2 family members. ${ }^{6}$ To examine the in vivo role of Bclaf1, we generated mice nullizygous for bclaf1 and discovered critical roles for Bclaf1 in lung development and proper functioning of the immune system. However, our findings do not support a role for Bclaf1 in regulating apoptosis in vivo.

Our histological analyses of bclaf1 ${ }^{-/-}$newborns uncovered a defect in lung development that explains the neonatal lethality of bclaf1 $1^{-1-}$ offspring. Lungs of bclaf1 ${ }^{-1}$ newborns were clearly defective in the proper formation of primitive alveoli or terminal air sacs that appear as open spaces on sections stained with hematoxylin and eosin. Events in murine lung formation are generally classified according to four developmental periods. The pseudoglandular stage (E9.5-16), characterized by formation of the bronchial tree, is followed by the canalicular stage $(E 16-17)$ that is characterized by the budding and branching of terminal epithelial tubules. These distal airspaces increase in size and number during the saccular stage (E17-PND5) through the process of primary septation. Type I and type II epithelial cells differentiate during this period and line the resulting saccules, which are embedded with a capillary network that facilitates gas exchange. The final alveolar stage is typified by secondary septation of primary saccules into mature alveoli, lined with type I and type II alveolar cells responsible for the processes of gas exchange and surfactant protein secretion. ${ }^{13}$ Our analysis of E16.5-PND1 lungs indicated that the developmental defect of bclaf1 mutants occurs during the saccular stage of development, as there was a defective or delayed thinning of the mesenchyme despite the presence of primitive alveoli in the mutant lungs. Although programmed elimination of cells through apoptotic processes coupled with proliferation arrest have been linked to end-stage lung development, the impaired lung development of Bclaf1-deficient mice appears independent of these processes. ${ }^{14}$ Our analyses of differentiation markers link the observed block in development to the inappropriate spatiotemporal accumulation of smooth muscle cells, as evident by the increased smooth muscle actin-positive cells in the Bclaf1-deficient lung mesenchyme at PND1. Furthermore, the persistence of expression of smooth muscle cell $\alpha$-actin in PND1 Bclaf1-deficient lungs suggests a critical role for Bclaf1 in pulmonary smooth muscle lineage development.

Our finding of polydactyly in bclaf1 $1^{-1-}$ mice implicates Bclaf1 in developmental processes independent of lung maturation. The production of supernumerary digits is normally suppressed during limb development through the restricted expression of the signaling molecule Sonic Hedgehog (Shh) to the posterior margin of the limb known as the zone of polarizing activity (reviewed in Lettice and Hill ${ }^{15}$ ). As several mouse mutants that exhibit misregulated expression of Shh during limb development or elevated fibroblast growth factor (Fgf) have increased incidence of polydactyly, it will be interesting to determine whether Bclaf1 participates in or modulates Shh or Fgf signaling. ${ }^{16-21}$

Given the role ascribed to Bclaf1 in apoptosis, we sought to determine whether Bclaf1 deficiency affects the cellular 
response to apoptotic stimuli. Furthermore, as Bcl-2 family members impact cell death processes in the context of immune system development and function, we examined the role of Bclaf1 in B and $T$ lymphocytes. To circumvent the perinatal lethality of $b c l a f 1^{-/-}$mice, lymphoid development was studied using wild-type, bclaf1 ${ }^{+/-}$, and $b c l a f 1^{-/-}$fetal livers to reconstitute lymphoid development in $\mathrm{V}(\mathrm{D}) \mathrm{J}$ recombinase-deficient $\operatorname{Rag}^{-/-}$recipients. Our studies show that Bclaf1 is dispensable for thymic development but is required for maintaining B- and T-cell homeostasis. In a bclaf1 mutant background, although thymocyte development was not affected, a drastic depletion of peripheral $T$ cells was observed in the spleen, LNs, and peripheral blood. In addition, a corresponding increase in the relative number of peripheral $B$ cells was also observed in the mutants. It is not clear whether the observed increase in the relative number of peripheral B cells is a direct result of Bclaf1 deficiency, or whether the increase is secondary to the observed depletion of peripheral T-cell numbers. Evaluation of the role of Bclaf1 in a given immune cell lineage would be facilitated by the generation of a conditionally disrupted Bclaf1 allele. In support of a reciprocity in B- and $\mathrm{T}$-cell homeostasis, earlier work in our laboratory, using mice with conditional disruption of genes in the T-cell lineage where a deficit in peripheral $\mathrm{T}$ cells is observed, has also observed a corresponding increase in peripheral $B$ cells in the spleen. ${ }^{22}$ Owing to the apparent deficit of peripheral $\mathrm{T}$ cells in Rag $^{-1-}$ mice reconstituted with bclaf $1^{-1}$ fetal liver cells, we examined the ability of these cells to proliferate in response to activation stimuli. Interestingly, deficiency of Bclaf1 results in impaired activation-driven proliferation of $T$ cells but not $B$ cells, and this deficit cannot be attributed to defects in apoptosis. Hence, Bclaf1 plays a critical role in lymphocyte homeostasis and activation.

Our results indicate that Bclaf1 is dispensable for execution of cell death pathways, suggesting that apoptosis reported earlier after transient overexpression of Bclaf1 may be a cellular response to the supraphysiological levels of Bclaf1. Nevertheless, a recent study has implicated Bclaf1 in the apoptotic response to DNA by inducing transcription of $p 53 .{ }^{23}$ A drastic depletion in Bclaf1 protein by RNAi was associated with an approximately two-fold reduction in apoptosis after doxorubicin exposure of U2OS cells that contain wild-type p53 but not in SaOS-2 cells deficient in p53. These results are in contrast with our findings, as Bclaf1-deficient thymocytes and splenocytes exposed to DNA-damaging agents that activate p53 ( $\gamma$-irradiation and etoposide) fail to show any defect in apoptosis compared with wild-type cells. Interestingly, the Bclaf1 protein species that was isolated on the basis of affinity for the core promoter element of p53 was $70 \mathrm{kDa}$, compared with the predicted molecular weights of 101 and $106 \mathrm{kDa}^{6}$ Further experimentation will need to be performed to clarify this discrepancy.

Given that the pleiotrophic phenotype in Bclaf1-deficient mice could not be explained by a corresponding deficit in apoptosis, we consider it unlikely that Bclaf1 is involved in cell death signaling in vivo. Two studies have linked Bclaf1 to a possible role in pre-mRNA processing. ${ }^{8,24}$ Bclaf1 contains an $\mathrm{RS}$ domain present in many pre-mRNA processing factors, and is present in interchromatin granule clusters, a subcellular structure that is enriched for such factors. ${ }^{8} \mathrm{~A}$ recent report identified Bclaf1 as a novel component of spliced and nonspliced ribonucleoprotein complexes. ${ }^{24}$ The association of Bclaf1 in such complexes was dependent on the presence of an m7G-cap-binding complex that contains the CBP80 and CBP20 cap-binding proteins. ${ }^{24}$ The $\mathrm{m} 7 \mathrm{G}$-cap has earlier been shown to be critical for pre-mRNA splicing, shuttling, nonsense-mediated decay, and protein translation. ${ }^{25,26}$ Whether defects in mRNA biogenesis play any role in mediating the pleiotrophic phenotype of Bclaf1-deficient mice requires further investigation.

\section{Materials and Methods}

Targeted disruption of bclaf1. A mouse $129 \mathrm{~Sv} / \mathrm{J}$ genomic library was screened with a mouse Bclaf1 cDNA probe. Overlapping clones spanning the 13-kb genomic region were found to contain four exons of the murine bclaf1 locus. A targeting strategy by the use of homologous recombination in ES cells was devised to delete an exon encoding $5^{\prime}$-untranslated sequence and the first coding exon of $b c l a f 1$ (Figure 1a). The neomycin gene in the targeting vector was flanked by a $6.0-\mathrm{kb}$ upstream homology region and a $0.6-\mathrm{kb}$ downstream homology region. Linearized targeting vector was electroporated into E14K ES cells (129 Sv/J background). G418-resistant ES cell clones were screened for homologous recombination by PCR using primers $5^{\prime}$-CACACTGCTCGATCCGGAACCCT- $3^{\prime}$ and $5^{\prime}$-CGACCCATTTCCAACAGAGCCAGAT-3'. Correctly targeted ES cells were verified by southern analysis using probes corresponding to the $3^{\prime}$-flanking region and the neomycin-coding region. Bclaf1 heterozygous ES cell lines were injected into C57BL/6 blastocysts and transferred to pseudopregnant ICR females. Chimeras were mated with C57BL/6 mice and germline transmission was obtained from two different ES clones. All data presented in this report were performed in both mutant mouse lines maintained in a mixed background (129 Sv/J and C57BL/6). Both mutant mouse lines were identical with respect to the observed phenotypes. All results presented were obtained from a minimum of three independent sibling pairs (wild-type and Bclaf1-deficient) derived from three independent pregnancies. Mice were maintained in accordance with the guidelines of the Animal Care Committee of the University Health Network Animal Facility. Routine genotyping of DNA was performed by PCR analysis using primers $5^{\prime}$-GACCCACCTCTTAGAAACTT- $3^{\prime}$ and $5^{\prime}$-CATCCACATAGACCAGCACT- $3^{\prime}$ for the wild-type allele or primers $5^{\prime}$-CCCAGCACCCGCCAGTAAGTCA-3 $3^{\prime}$ and $5^{\prime}$-TAC CCTCTACCCCTTCCTCTGTA- $3^{\prime}$ for the mutant allele. Embryonic fibroblasts were derived from wild type, and Bclaf1 homozygous E15.5 embryos were prepared according to standard procedures.

Northern analysis. Total RNA from wild-type, bclaf1 ${ }^{+/}$, and $b c l a f 1^{-1-}$ embryonic fibroblasts was fractionated on a $1.2 \%$ formaldehyde agarose gel and transferred to Hybond $\mathrm{N}^{+}$membrane (Amersham). Hybridization was performed using a ${ }^{32} \mathrm{P}$-random-primed labeled 2.8-kb Bclaf1 $\mathrm{CDNA}$ probe comprising the entire open reading frame.

Western analysis and indirect immunofluorescence. A rabbit polyclonal antibody raised against mouse Bclaf1 amino acid residues 470-483 (AKDKHKEEDKGSDR) was generated and affinity purified (Open Biosystems). Wild-type and bclaf1 ${ }^{-/-}$embryonic fibroblasts were lysed in $20 \mathrm{mM}$ HEPES pH 8.0, $15 \mathrm{mM} \mathrm{MgCl}$, $25 \%$ glycerol, $420 \mathrm{mM} \mathrm{NaCl}, 2 \mathrm{mM}$ EDTA, and $1 \mathrm{mM}$ DTT (supplemented with Protease Inhibitor Cocktail, Sigma). Lysates were sonicated for $5 \mathrm{~s}$ on ice and cleared by centrifugation $\left(14000 \times g\right.$ for $\left.10 \mathrm{~min}, 4^{\circ} \mathrm{C}\right)$. Supernatants $(60 \mu \mathrm{g})$ were resolved by SDS-PAGE, transferred onto the PVDF membrane (Pall Life Sciences), and immunoblotted under standard conditions with anti-Bclaf1 antibody followed by anti-actin (Sigma) to ensure equal loading. Anti-mouse or antirabbit IgG HRP-linked secondary antibodies and chemiluminescence detection reagents were from GE Healthcare and Pierce, respectively. Indirect immunofluorescence using anti-Bclaf1 (no. 1704, Novus) was performed using wild-type and bclaf1 ${ }^{-1-}$ fibroblasts seeded on coverslips and fixed using cold methanol/acetone. Coverslips were then stained with donkey anti-rabbit FITC secondary antibody (Jackson), mounted with Vectashield with DAPI (Vector), and visualized using a Zeiss Axioplan 2.0 microscope and Axiovision software (Zeiss).

Expression analysis of genes proximal to the targeted Bclaf1 locus by quantitative real-time PCR. First-strand cDNA was prepared 
from $2 \mu \mathrm{g}$ of total RNA from wild-type and bclaf1 ${ }^{-1-}$ fibroblasts using Superscript III reverse transcriptase (Invitrogen). Quantitative PCR was performed in triplicate assays using the Power SYBR Green PCR master mix (Applied Biosystems) for each target gene as well as an internal control GAPDH. PrimerExpress (Applied Biosytems) software was used to design the following primers: 2610016C23riken sense primer $5^{\prime}$-GCCAGAGACTTCTGATGTCC-3', 2610016C23riken antisense primer $5^{\prime}$-TTCTGAACGGGTCTGC-3', Mtap7 sense primer $5^{\prime}$-GGAGGAC AGAGACCGCTGATA-3', and Mtap7 antisense primer $5^{\prime}$-CACGGCAGTGCA GGTACTTC-3'. GAPDH sense primer $5^{\prime}$-GGGCATCTTGGGCTACACTG- $3^{\prime}$ GAPDH antisense primer $5^{\prime}$-AGCCGTATTCATTGTCATACC-3'. Reactions were performed in a $25 \mu \mathrm{l}$ final mixture containing $12.5 \mu \mathrm{l}$ PCR master mix, $2.5 \mu \mathrm{l}$ CDNA, $25 \mathrm{pmol}$ of each primer, and $9.5 \mu \mathrm{l}$ water. PCR amplification reactions were performed in a 7500 Real-Time PCR System (Applied Biosystems) as follows: 40 cycles with denaturation at $95^{\circ} \mathrm{C}$ for $15 \mathrm{~s}$, annealing at $50^{\circ} \mathrm{C}$ for $2 \mathrm{~min}$, and extension at $60^{\circ} \mathrm{C}$ for $1 \mathrm{~min}$. The expression of target genes was normalized by the internal reference gene, GAPDH. A no-template negative control was also included in all experiments. Each reaction was subjected to melting point analysis to confirm single amplified products. Moreover, a control cDNA dilution series was created for each gene to establish a standard curve and to check the PCR efficiency. Fold changes in gene expression were determined using the $2^{-\Delta \Delta C_{\mathrm{t}}}$ method.

Histology. Embryos and tissues from neonates were fixed in formalin, and paraffin sections were stained with hematoxylin and eosin according to standard procedures. For measurement of BrdU incorporation, pregnant mice were injected with $0.1 \mathrm{mg}$ BrdU per gram mouse weight intraperitoneally $45 \mathrm{~min}$ before embryo collection. Sections of embryos were immunostained for BrdU as described earlier. ${ }^{27}$ Monoclonal antibody to PCNA (Santa Cruz Biotechnology, Santa Cruz, CA, USA) (dilution $1: 1000$ ), rabbit polyclonal antibodies against cleaved caspase 3 (Cell signaling) (dilution 1:200), and TUNEL reagent (Roche) were also used. Sections stained for BrdU, PCNA, TUNEL, or cleaved caspase 3 were counterstained with 4',6-diamidino-2-phenylindole dihydrochloride (DAPI), and three photomicrographs per section were captured using the same exposure settings for a given experiment using a Zeiss Axioplan 2.0 microscope and Axiovision software (Zeiss). For quantitative measurements of proliferation (PCNA and BrdU) or apoptosis (cleaved caspase 3), 1000 cells were scored as either PCNA, BrdU, or cleaved caspase 3/TUNEL positive from three photomicrographs per section by an observer blind to the genotype from three sibling sets of wild-type and $b c l a f 1^{-1-}$ lungs. For detection of specific cell lineages, the following antibodies were used: hamster monoclonal anti-mouse $\mathrm{T} 1-\alpha$ (Developmental Studies Hybridoma Bank, University of lowa, Hybridoma no. 8.1.1) (1:50 dilution), mouse monoclonal anti-mouse $\alpha$-smooth muscle actin ( $\alpha$-sma) (Labvision/ Neomarkers, Fremont, CA, USA) (1:1000 dilution), rabbit polyclonal antibodies against pro-N-SP-C (1:500 dilution) generously provided by Dr. M Beers (University of Pennsylvania, Philadelphia, PA, USA), and rabbit polyclonal antibodies against CCSP (1: 1000 dilution), a gift from Dr. SL Kaytal (Pittsburgh, PA, USA).

Fetal liver transfer. For generation of $b \mathrm{claf}^{-1-} \mathrm{Rag}^{-1-}$ chimeras, fetal liver suspensions $\left(5 \times 10^{6}\right.$ cells $)$ from bclaf1 ${ }^{-1-}$ embryos or littermates with heterozygote or wild-type genotype were i.v. injected into irradiated Rag2 ${ }^{-1-}$ recipients ( 3 Gy). Lymphoid reconstitution was analyzed in the thymus, spleen, LNs, and blood 4-5 weeks later.

Flow cytometric analysis. Single cell suspensions from the thymus, spleen, and LNs were stained and analyzed by flow cytometry using a FACScan (Becton Dickinson). All antibodies were purchased from PharMingen.

Analysis of lymphocyte survival and proliferation. Lymphocytes $\left(1 \times 10^{5}\right)$ were left untreated or exposed to drugs for $24 \mathrm{~h}$ before harvesting. Apoptosis was evaluated by flow cytometry (FACScan, Becton Dickinson) after staining with Annexin V-FITC and propidium iodide (R\&D systems). Analysis of proliferation and cell cycle after activation was performed essentially as described in McPherson. ${ }^{28}$

Acknowledgements. We thank Eileen White and Minna Woo for helpful discussions and also thank Denis Bouchard and Drew Wakeham for technical assistance. This study was supported by grants from the Canadian Institute of Health Research (MOP 36537) and the National Cancer Institute of Canada (TFPP
12000) to $\mathrm{RH}$, and from the Canadian Institutes of Health Research (MOP 74674) and the Canadian Lung Association/Ontario Thoracic Society to JPM. JPM and RH are both supported by salary awards from the Canadian Institute of Health Research.

1. Danial NN, Korsmeyer SJ. Cell death: critical control points. Cell 2004; 116: 205-219.

2. Johnstone RW, Ruefli AA, Lowe SW. Apoptosis: a link between cancer genetics and chemotherapy. Cell 2002; 108: 153-164.

3. Adams JM, Cory S. The Bcl-2 protein family: arbiters of cell survival. Science 1998; 281: 1322-1326

4. Huang DCS, Strasser A. BH3-only proteins - essential initiators of apoptotic cell death. Cell 2000; 103: 839-842.

5. Cory S, Adams J. The Bcl-2 family: regulators of the cellular life-or-death switch. Nat Rev 2002; 2: 647-656.

6. Kasof GM, Goyal L, White E. Btf, a novel death-promoting transcriptional repressor that interacts with bcl-2-related proteins. Mol Cell Biol 1999; 19: 4390-4404.

7. Valcarcel J, Green MR. The SR protein family: pleiotropic functions in pre-mRNA splicing. Trends in Biochem Sci 1996; 21: 296-301.

8. Saitoh N, Spahr CS, Patterson SD, Bubulya P, Neuwald AF, Spector DL. Proteomic analysis of interchromatin granule clusters. Mol Biol Cell 2004; 15: 3876-3890.

9. Wilkinson E, Holaska J, Zhang Z, Sharma A, Manilal S, Holt I et al. Emerin interacts in vitro with the splicing-associated factor, YT521-B. Eur J Biochem 2003; 270: 2459-2466.

10. Haraguchi T, Holaska JM, Yamane M, Koujin T, Hashiguchi N, Mori C et al. Emerin binding to $\mathrm{Btf}$, a death-promoting transcriptional repressor, is disrupted by a missense mutation that causes Emery-Dreifuss muscular dystrophy. Eur J Biochem 2004; 271: 1035-1045.

11. Komada M, McLean DJ, Griswold MD, Russell LD, Soriano P. E-MAP-115, encoding a microtubule-associated protein, is a retinoic acid-inducible gene required for spermatogenesis. Genes Dev 2000; 14: 1332-1342.

12. Ten Have-Opbroek AA. Lung development in the mouse embryo. Exp Lung Res 1991; 17: 111-130.

13. Maeda Y, Dave V, Whitsett JA. Transcriptional control of lung morphogenesis. Physiol Rev 2007; 87: 219-244.

14. Sutherland HGE, Mumford GK, Newton K, Ford LV, Farrali R, Dellaire G et al. Large-scale identification of mammalian proteins localized to nuclear sub-compartments. Hum $\mathrm{Mol}$ Genet 2001; 10: 1995-2011.

15. Lettice LA, Hill RE. Preaxial polydactyly: a model for defective long-range regulation in congenital abnormalities. Curr Opin Genet Dev 2005; 15: 294-300.

16. Sharpe J, Lettice L, Hecksher-Sorensen J, Fox M, Hill R, Krumlauf R. Identification of Sonic hedgehog as a candidate gene responsible for the polydactylous mouse mutant Sasquatch. Curr Biol 1999; 9: 97-100.

17. Clark RM, Marker PC, Kingsley DM. A novel candidate gene for mouse and human preaxial polydactyly with altered expression in limbs of Hemimelic extra-toes mutant mice. Genomics 2000; 67: 19-27.

18. Litingtung Y, Dahn RD, Li Y, Fallon JF, Chiang C. Shh and Gli3 are dispensable for limb skeleton formation but regulate digit number and identity. Nature 2002; 418: 979-983.

19. Sagai T, Masuya H, Tamura M, Shimizu K, Yada Y, Wakana S et al. Phylogenetic conservation of a limb-specific, cis-acting regulator of Sonic hedgehog (Shh). Mamm Genome 2004; 15: 23-34.

20. Adamska M, MacDonald BT, Meisler MH. Doubleridge, a mouse mutant with defective compaction of the apical ectodermal ridge and normal dorsal-ventral patterning of the limb. Dev Biol 2003; 255: 350-362.

21. Hajihosseini MK, Lalioti MD, Arthaud S, Burgar HR, Brown JM, Twigg SR et al. Skeletal development is regulated by fibroblast growth factor receptor 1 signaling dynamics. Devel 2004; 131: 325-334.

22. Salmena L, Lemmers B, Hakem A, Matysiak-Zablocki E, Murakami K, Au PYB et al. Essential role for caspase 8 in T-cell homeostasis and T-cell-mediated immunity. Genes Dev 2003; 17: 883-895.

23. Liu H, Lu Z-G, Miki Y, Yoshida K. Protein kinase $C$ delta induces transcription of the TP53 tumor suppressor by controlling death-promoting factor Btf in the apoptotic response to DNA damage. Mol Cell Biol 2007; 27: 8480-8491.

24. Merz $\mathrm{C}$, Urlaub H, Will CL, Luhrmann R. Protein composition of human mRNPs spliced in vitro and differential requirements for mRNP protein recruitment. RNA 2007; 13: 116-128.

25. Lewis JD, Izaurralde E. The role of the cap structure in RNA processing and nuclear export. Eur J Biochem 1997; 247: 461-469.

26. Hosoda N, Kim YK, Lejene F, Maquat LE. CBP80 promotes interaction of Up1 with Upf2 during nonsense-mediated mRNA decay in mammalian cells. Nat Struc Mol Biol 2005; 12 : 893-901.

27. McPherson JP, Tamblyn L, Elia A, Migon E, Shehabeldin A, Matysiak-Zablocki E et al. Lats2/Kpm is required for embryonic development, proliferation control and genomic integrity. The EMBO J 2004; 23: 3677-3688.

28. McPherson JP, Lemmers B, Hirao A, Hakem A, Abraham J, Migon E et al. Collaboration of Brca1 and Chk2 in tumorigenesis. Genes Dev 2004; 18: 1144-1153. 